\title{
Zur Theorie des staatsmonopolistischen Kapitalismus
}

\author{
Robert Katzenstein*
}

Bei der Theorie des staatsmonopolistischen Kapitalismus handelt es sich um eine Analyse der Entwicklung des Kapitalismus in der Gegenwart, ihrer Erscheinungen und Gesetzmäßigkeiten. Das ist ein weites Feld, es läßt sich nicht in kurzen Worten abhandeln. Die Theorie ist auch weder in sich geschlossen, in dem Sinne, daß auf alle Fragen bereits eine Antwort gefunden wurde, noch erhebt sie Anspruch, daß alle Antworten, die sie schon geben kann - und das sind nicht wenige - auch bereits der Erkenntnis letzter Schluß, also völlig befriedigend sind. Es gibt unter marxistischen ökonomen sehr unterschiedliche Ausgangspunkte und sehr unterschiedliche Auffassungen hinsichtlich der Erklärung bestimmter Erscheinungen und Gesetzmäßigkeiten. Ich muß mich hier also darauf beschränken, einige wesentliche Grundzüge dieser Theorie zu umreißen.

Zunächst zur Bildung des Begriffs staatsmonopolistischer Kapitalismus. Ausgangspunkt der Untersuchung war die starke Zunahme der staatlichen Eingriffe in die Wirtschaft nach dem II. Weltkrieg. Sie wurden zunächst mit den Besonderheiten der Nachkriegsentwicklung erklärt. Im Verlauf der Untersuchung zeigte sich dann, $\mathrm{da}$ es sich keineswegs um aus Besonderheiten herrihrende Eingriffe handelte - obwohl diese die auslösenden Faktoren bildeten - sondern daß sie notwendig aus den Konflikten selbst entsprangen, die die kapitalistische Bewegung der gesellschaftlichen Produktion hervorruft. Sie waren gesetzmäßig entstanden und sie hatten Systemcharakter, d.h. sie waren gesetzmäßig zum Bestandteil der Bewegung des Kapitalismus der Gegenwart geworden. Da es sich bei diesem Kapitalismus um Monopolkapitalismus handelt und die staatlichen Eingriffe auf das Funktionieren dieses Systems gerichtet waren, lag es nahe, diese neuen Erscheinungen im Begriff staatsmonopolistischer Kapitalismus zusammenzufassen. Der weitere Verlauf der Untersuchung ergab dann, daß es sich bei diesen staatlichen Eingriffen, ihrem Charakter nach, um Monopolbedingungen handelte, die der Staat in der Wirtschaft setzte, d. h. um Bedingungen, die gegen das Wirken des Konkurrenzmechanismus gerichtet waren und die für sein Wirken bestimmte neue Bedingungen setzten. Es handelt sich um eine höhere Form der Monopolisienung. Sie beeinflußt, wie das ökonomische Monopol auch, die Verteilung des Nationaleinkommens entgegen der sich aus den inneren Gesetzmäßigkeiten des Kapitalismus ergebenden Verteilung. Von Staats wegen wurden hier Bedingungen in der Wirtschaft gesetzt, die die Verwertung des Kapitals beeinflußten. Die ökonomische Macht der Monopole wurde auf diese Weise durch die außerökonomische Macht des Staates erweitert; und dies

*) Die Beiträge von R. Katzenstein und M. Wirth sind Referate, welche auf der konstituierenden Sitzung des Arbeitskreises Politlsche ökonomie der Deutschen Vereinigung für Politische Wissenschaft am 11. Mai 1973 im Fachbereich 15 (Otto-Suhr-Institut) der Freien Universität Berlin gehalten wurden und hier unverändert wiedergegeben werden. 
war notwendig, um die Bewegung der gesellschaftlichen Produktion als kapitalistische Produktion überhaupt zu ermöglichen.

An sich liegt es durchaus nahe, daß das Monopolkapital, sobald es zur ökonomischen Grundlage der gesellschaftlichen Produktion geworden ist, sich der Macht des Staates zu bedienen sucht, um seine Verwertung zu steigern. Derartige Erscheinungen sind nicht neu und sie werden immer wieder auch an der Oberfläche in ganz eindeutiger Form sichtbar; man denke hier nur an die Herrschaftsformen amerikanischer Konzerne in Südamerika, an die englische East India Company oder die Beziehungen zwischen den IG-Farben und der Nazi-Regierung. Auch in jüngster Zeit gibt es dafür genügend Beispiele.

Zur Gesetzmäßigkeit einer Entwicklung gehört jedoch mehr, als nur der Wille und die Macht der Beteiligten, dazu gehört auch die Notwendigkeit solcher Entwicklungen.

Untersucht man die Bedingungen, denen der Kapitalismus heute unterworfen ist, denen er in seiner Entwicklung Rechnung tragen muß, so schält sich diese Notwendigkeit sehr eindeutig heraus und man kann ihre Wurzeln sogar bis in die Anfänge dieses Jahrhunderts hinein verfolgen. Ganz allgemein gesehen, entspringt sie aus der Zuspitzung des kapitalistischen Grundwiderspruches zwischen dem gesellschaftlichen Charakter von Produktion und Produktivkräften und den durch die private Aneignung der Produkte dieser gesellschaftlichen Produktion charakterisierten Produktionsverhältnissen. Konkreter besehen, äußert sich dieser Widerspruch in zwei großen Komplexen:

Erstens in den Bedingungen, denen die Kapitalbewegung selbst durch die technische Revolution unterworfen wird. Der Prozeß der Kapitalakkumulation wird von der Seite der Entwicklung der Produktivkräfte her Bedingungen unterworfen, die gegenüber früher zwar keine neuen, aber doch von ihrer Bedeutung her qualitativ veränderte Anforderungen stellen. Der Grundwiderspruch tritt ganz unmittelbar in der Form in Erscheinung, daß das Kapitalverhältnis zu einer Schranke für die Entwicklung der Produktivkräfte wird. Und damit entsteht auch eine Schranke für die Kapitalbewegung selbst. Staatliche Eingriffe werden notwendig, um Raum für diese Bewegung und für die Entwicklung der Produktivkräfte auf kapitalistischer Grundlage zu schaffen.

Zweitens hat der Grundwiderspruch, vermittelt über andere Konflikte, über Krisen und Kriege, auch in anderer Hinsicht neue Bedingungen geschaffen. Auch diese sind nicht absolut neu, aber von qualitativ neuer Bedeutung. Seit der sozialistischen Revolution in Rußland haben die inneren, sozialen Konflikte des Kapitalismus einen Reifegrad erlangt, daß sie seine Existenz selbst in Frage stellen. Seither stellen sich diese inneren Konflikte auch äußerlich als Gegensatz zwischen Kapitalismus und Sozialismus dar. Seither wird der Kapitalismus, ganz gleich in welchen Formen sich das äußert und ob im Innem der hochindustrialisierten kapitalistischen Länder oder in ihrer äußeren Einflußsphäre, am Sozialismus gemessen, d. h. seine Bewegungsfreiheit ist eingeengt. Und in dieser Hinsicht sind nach dem II. Weltkrieg ebenfalls Bedingungen neuer Qualität entstanden; durch die Bildung des sozialistischen Systems; durch den Zerfall des Kolonialsystems; durch die Entfaltung demokratischer Massenbewegungen in den imperialistischen Ländern selbst, die von ih- 
rem Umfang her, von der Breite der erfaßten Schichten, von deren Organisiertheit und, bei allen Unterschieden in den Auffassungen im einzelnen, von ihrer antimonopolistischen Zielstellung her eine Kraft neuer Qualität darstellen. Das alles sind Bedingungen, denen der Kapitalismus in seiner Bewegung heute Rechnung tragen muß. Es ist unmöglich, daß er den Konflikten, die aus dem inneren Wesen des Kapitals und aus seiner spontanen Bewegung heraus entstehen, freien Lauf läßt, ohne daß er das System selbst in Gefahr bringt. Auch aus diesen Gründen sind staatliche Eingriffe zur Regulierung der Produktion unerläßlich geworden.

Beide Ursachenkomplexe sind wechselseitig verbunden, sie beeinflußen sich gegenseitig; aber darauf sei hier nur hingewiesen.

Politische Gründe verursachten bereits die ersten, wirklich großangelegten ökonomischen staatsmonopolistischen Eingriffe im Zusammenhang mit der Weltwirtschaftskrise von 1929/33. Ihre Wurzeln liegen in den Klassenkämpfen nach dem I. Weltkrieg, die das gesellschaftliche System des Kapitalismus erschütterten und die in der Krise erneut und mit großer Heftigkeit aufflammten. Die weltweite Krise war von einer bislang unbekannten Schärfe und ihr folgte eine langandauernde Stagnation. Die Massenarbeitslosigkeit, die Verelendung weiter Schichten der Bevölkerung und zugleich auch die Ausweglosigkeit der Situation führten zu sozialen Unruhen und politischen Krisen und Kämpfen, wie man sie vorher in Friedenszeiten noch nicht erlebt hatte. Der Staat mußte eingreifen. Und zwar nicht nur politisch, durch den Übergang zum Faschismus, sondern auch ökonomisch, um die ökonomischen Grundlagen der sozialen Unruhen zu beseitigen. In der Hauptsache erfolgten die Eingriffe damals über staatliche Investitionen; sie stiegen in den folgenden Jahren sprunghaft an und machten 1935 rund 44 Prozent der volkswirtschaftlichen Gesamtinvestitionen aus, gegenüber 20 Prozent im Durchschnitt der Aufschwungsjahre von 1924 bis 1928 .

Weder die Tiefe der Krise, noch die Dauer der Stagnation, noch die Verbindung der staatlichen Eingriffe mit den Investitionsprozessen waren zufällig. Die Investitionsprozesse spielen als materielle Basis der zyklischen Produktionsbewegungen im Kapitalismus eine zentrale Rolle. Gerade die Investitionen aber hatten im Laufe der Entwicklung ihre Bedeutung im Rahmen des Gesamtablaufs der volkswirtschaftlichen Prozesse ganz beträchtlich gesteigert. 1925 war bereits jeder vierte Beschäftigte in der deutschen Volkswirtschaft mit der Produktion von Teilen des fixen Kapitals befaßt; nach 1875 war es erst etwa jeder neunte gewesen. Man kann sich also leicht vorstellen, welche Auswirkungen der Rückgang der Investitionen um rund 66 Prozent - über die mit ihm verbundene Multiplikatorwirkung - auf die Tiefe der Krise haben mußte. Und auch weiterhin haben muß! Seitdem hat das relative Gewicht des fixen Kapitals noch zugenommen. 1962 war bereits jeder dritte Beschäftigte in der Volkswirtschaft der Bundesrepublik mit der Produktion von Arbeitsmitteln befaßt. Seit der Weltwirtschaftskrise ist daher die Gefahr, daß Uberproduktionskrisen sich zu außerordentlich tiefen und für die politische Stabilität des Systems gefährlichen Krisen ausweiten, sehr groß und seit damals spielen daher auch Maßnahmen zu ihrer Verhinderung oder Abschwächung in der staatlichen Wirtschaftspolitik eine ganz entscheidende Rolle; und sie sind erklärlicherweise auch hauptsächlich mit den volkswirtschaftlichen Investitionsprozessen verbunden. 
Man kann sagen, daß die Absicherung der Existenz des kapitalistischen Systems seit der Weltwirtschaftskrise auch zu einer ökonomischen Funktion des bürgerlichen Staates geworden ist.

Die Tiefe der Krise war jedoch nicht nur durch das relative Gewicht des fixen Kapitals bedingt. Auch das Monopol hat dazu beigetragen. Anders als im einfachen Kapitalismus, da alle Kapitale unter dem Druck der Konkurrenz weiter produzieren müssen bis sie untergehen, selbst bei enormem Preisdruck, legt das Monopol alle Betriebe still, mit Ausnahme derjenigen, die ihm unter den gegebenen Marktverhältnissen und bei entsprechenden Preisabsprachen eine optimale Verwertung verspre. chen. Der Umfang der Stillegungen und die Senkung des Verbrauchs sind daher größer als im vormonopolistischen Kapitalismus. Die Weltwirtschaftskrise war also eine für den Monopolkapitalismus typische Krise.

Auch die Dauer der Stagnation nach dieser Krise erklärt sich aus dem Monopol. Das einfache Kapital kann auf die durch die Krise veränderten Konkurrenzund Verwertungsbedingungen nur auf eine Weise reagieren: durch die Modernisierung seiner Produktionsanlagen. Auch das Monopol reagiert auf diese Weise, aber es beschränkt die Modernisierung auf die Anlagen, die es in Betrieb hält, d. h. es engt den Umfang der Investitionsprozesse ein und begrenzt so den Einfluß, der von ihnen auf den Produktionsaufschwung ausgeübt werden kann. Die Reaktion des Kapitals auf die veränderten Bedingungen löst also nicht mehr automatisch den auf die Krise relativ bald folgenden Aufschwung aus.

Bei der Weltwirtschaftskrise handelt es sich also um eine für den Monopolkapitalismus typische Krise. Seit damals ist die Gefahr, daß sich die periodischen Überproduktionskrisen zu tiefen, langandauemden und für die politische Stabilität des Systems gefährlichen Krisen ausweiten, außerordentlich groß. Seit damals spielen daher auch Maßnahmen zu ihrer Verhinderung oder Begrenzung in der staatlichen Wirtschaftspolitik eine erstrangige Rolle; und sie sind erklärlicherweise auch hauptsächlich mit den volkswirtschaftlichen Investitionsprozessen verbunden. Man kann sagen, daß seit der Weltwirtschaftskrise die Sicherung der Existenz des kapitalistischen Systems auch zu einer ökonomischen Funktion des bürgerlichen Staates geworden ist.

Obwohl nach dem II. Weltkrieg, bedingt durch die Veränderungen im politischen Gefüge der Welt und namentlich im Kräfteverhältnis zwischen Kapital und Arbeit, einige Faktoren ohnehin dahin wirken, die Vertiefung der Krisen zu begrenzen $-z$. B. ist es für das Kapital unmöglich geworden, die Lasten solcher Krisen voll oder auch nur mittelfristig auf die Arbeiter abzuwälzen - spielt diese Frage der Regulierung der Produktionsbewegung nach wie vor eine ganz zentrale Rolle. Sie hat sogar insofern noch an Bedeutung gewonnen, als das Kapitalverhältnis in zunehmendem Maße zu einer Schranke für die Entwicklung der Produktivkräfte zu werden beginnt. Die Investitionsprozesses des privaten Kapitals laufen daher periodisch Gefahr, ins Stocken zu geraten und den Umschlag der Produktionsbewegung in eine Krise auszulösen. Die staatliche Konjunkturpolitik, die inzwischen sogar notwendigerweise zu einer Wachstumspolitik geworden ist, gewinnt daher heute größere Bedeutung denn je. Gleichzeitig hat sich aber das Schwergewicht der Problematik verschoben. Die staatlichen Eingriffe können sich nicht mehr darauf beschränken, 
die Bewegungsform der kapitalistischen Produktion zu regulieren, sondern sie müssen schon auf die Bewegung des Kapitals selbst gerichtet werden, müssen Schranken durchbrechen, die seinen Bewegungsspielraum zunehmend einengen.

Auf diese Problematik möchte ich jetzt eingehen, allerdings nur auf eine ihrer Seiten. Ich sagte eingangs, daß sich der Grundwiderspruch zwischen dem gesellschaftlichen Charakter der Produktivkräfte und den kapitalistischen Produktionsverhältnissen nach dem II. Weltkrieg derart zugespitzt hat, daß das Kapitalverhältnis immer offener und eindeutiger als Schranke für die Entwicklung der Produktivkräfte hervortritt. Kernproblem ist hier das rapide Wachstum des fixen Kapitals, das von seinem Umschlag her ganz neue Anforderungen an den Vergesellschaftungsgrad der Produktion stellt, Anforderungen, die gesellschaftliche Organisation der Produktion notwendig machen und denen die kapitalistische Aufteilung der Produktion widerspricht. Ich spreche von Aufteilung nicht von Zersplitterung, weil dieser Begriff die heutigen Bedingungen hochkonzentrierter Produktion besser kennzeichnet, obwohl er andererseits das Moment der Privatinteressen, ihrer Gegensätzlichkeit, der Konkurrenz etc. eher etwas verwischt.

Die Entwicklung der modemen Technik wird durch ein sprunghaftes Wachstum der Kapazitäten charakterisiert. In der Regel wächst dabei das fixe Kapital überproportional. Von seinem Umschlag her gesehen, ergibt sich daher ein Zwang zu beträchtlicher Absatzsteigerung oder, anders ausgedrückt, ein Zwang zur Kontinuität der Produktion auf hoher gesellschaftlicher Stufenleiter. Anders läßt sich die moderne Technik nicht rentabel ausnutzen. Das gilt nebenbei selbst für den arlagekapitalsparenden Typ der Technik.

In der Mineralölindustrie, beispielsweise, sind die Investitionskosten je Tonne Jahreskapazität gegenüber der Vorkriegszeit auf etwa ein Viertel gesunken. Der absolute Investitionsaufwand für optimale A nlagen ist jedoch derart gestiegen, daß sich der Absatz mehr als verdreifachen mußte, wenn der Verschleißteil von fixem Kapital je Erzeugniseinheit gleichbleiben sollte. Er mußte sich mehr als verzehnfachen, sobald die neue Technik zur allgemeinen technologischen Basis der Raffinerieproduktion geworden war und folglich die Konkurrenzverhältnisse bestimmte. Die Mineralölverarbeitung ist dabei noch eine Ausnahme. In den meisten Zweigen sind die Investitionskosten je Tonne Jahreskapazität gestiegen und zwar zum Teil beträchtlich. Je schneller also das relative Gewicht des fixen Kapitals wächst, um so mehr wird die Auslastung der Anlagen zu einer wesentlichen Bedingung der Kapitalverwertung. Der Zwang zur Kontinuität der Produktion auf hoher gesellschaftlicher Stufenleiter verstärkt sich ungemein.

Es kommt noch ein weiteres Moment hinzu: das der Vertiefung der Arbeitsteilung und der engeren und unmittelbareren Verflechtung der verschiedenen Produktionsstufen. Die Proportionalität der Produktionsstufen, eine wichtige Bedingung der Kapitalverwertung, setzt ebenfalls neue Maßstäbe in Bezug auf die Größenordnungen der Gesamtanlagen. Es sei nebenbei erwähnt, daß dieses Problem sich gesamtvolkswirtschaftlich als Proportionalität der volkswirtschaftlichen Bereiche niederschlägt und auch in dieser Form gesamtgesellschaftlichèr Produktionszusammenhänge in ganz neuen Maßstäben zu einer Voraussetzung rationeller Anwendung moderner Produktivkräfte wird. Man kann sich das in seiner Bedeutung leicht sicht- 
bar machen, wenn man überdenkt, in was für ein Netz von volkswirtschaftlichen Beziehungen Unternehmen der neuen Größenordnungen reibungslos eingebettet sein müssen, wenn sie profitabel arbeiten sollen. Jedoch zurück zu den Unternehmungsgrößenordnungen.

Vergleicht man beispielsweise die in der Eisen- und Stahlindustrie noch Anfang der 5Ger Jahre angewandte Technik mit dem Stand, der die darauf folgende technische Entwicklungsstufe charakterisierte, so zeigt sich ebenfalls ein sprunghaftes Wachstum der Kapazitäten der Aggregate in den einzelnen Produktionsstufen. Die Tagesleistung der Hochöfen hat sich mehr als verzehnfacht; das Chargengewicht der Siemens-Martin-Öfen hat sich etwa verfünfacht, wobei die Schmelzzeit der Chargen überdies auf ein Drittel verkürzt wurde, die Leistung der Walzenstraßen ist etwa auf das Fünfundzwanzigfache gestiegen.

Schon diese wenigen Beispiele zeigen, daß jede Veränderung in einem Teilbereich die Proportionalität der Gesamtanlage sprengt, von der das reibungslose Ineinandergreifen der einzelnen Produktionsbereiche und folglich die Rentabilität der Gesamtanlage abhängt. Die technische Revolution erzwingt also die völlige Umgestaltung eines Hüttenwerkes. Noch etwas hat sich geändert. Im Gegensatz zu früher sind heute die Walzwerke strukturbestimmend. Mißt man die Größenordnung eines von der technischen Zusammensetzung der Anlagen her optimal strukturierten Hüttenwerkes an der jährlichen Rohstahlproduktion, so betrug sie bei den alten Anlagen rund $800000 \mathrm{t}$, bei den neuen liegt sie bei etwa 5 Mio. t.

Soweit die unmittelbare produktionsmäßige Verflechtung. Für einen Konzern tritt aber noch die Frage seiner Stellung im Konkurrenzkampf hinzu. Eine optimale Konzernstruktur, d. h. eine solche, die dem Konzern von der Breite des Produk. tionssortiments und zugleich von der Proportionalität der Anlagen her, eine feste Stellung im Markt verleiht und die früher ebenfalls bei rund $800000 \mathrm{t} \mathrm{lag}$, liegt heute bei 10 bis 12 Mio.t.

Dabei zeigt sich ein Wesenszug kapitalistischer Produktionsverhältnisse, der in diesem Zusammenhang bedeutsam ist. Ein Konzern kann sich nicht einfach in gesellschaftliche Produktionszusammenhänge einordnen. Er kann sich nicht darauf beschränken, um ein Beispiel zu nennen, durch Spezialisierung den rationellen Einsatz moderner Technik in der optimalen Größenordnung eines Hüttenwerkes von 5 Mio. $t$ zu ermöglichen - einer Größenordnung, die Mitte der 50er Jahre von einer. Reihe von Unternehmen im Zuge des Akkumulationsprozesses hätte erreicht werden können - sondern er muß seine Stellung im Konkurrenzkampf, seine relative Machtposition im Gefüge des gesellschaftlichen Gesamtkapitals dadurch $v^{-6}$ ien, daß er alle Hauptstrukturen seines Produktionsbereiches umfaßt. D. h. die G. iß ordnungen, die ein Konzern erreichen muß, um die Potenzen der neuen Ttwhrik ausschöpfen zu können, werden nicht nur technisch, sondern auch durch die Notwendigkeit der Aufrechterhaltung seiner Monopolstellung bestimmt; sie vervielfachen sich dadurch erneut. Und in dieser Hinsicht verändern sich die Bedingungen ebenso schnell wie durch die rein technisch bedingte Entwicklung. Noch vor zehn Jahren waren in der Eisen- und Stahlindustrie die Walzwerke strukturbestimmend. Heute ist die gesellschaftliche Stufenleiter der Produktion bereits so weit fortgeschritten, daß Konzernstrukturen auch in diesem Bereich die Weiter- bzw. Endverar- 
beitung mit einbeziehen müssen, wenn das gesamte notwendige Netz einer Monopolstruktur erfaßt werden soll. Es sind Imperien volkswirtschaftlicher Größenordnungen, die sich da herausbilden müssen, wenn der erforderliche Vergesellschaftungsgrad der Produktion erreicht werden soll, der sowohl die technischen Gesichtspunkte als auch die des Monopols beriicksichtigt. Der Vergesellschaftungsgrad der Produktion wird durch die Entwicklung der Produktivkräfte also ganz enorm gesteigert und er ist, im Gegensatz zu früher, heute nicht mehr nur die Folge, sondern mehr und mehr schon die Voraussetzung für die rationelle Anwendung moderner Technik.

Früher spielte es für die Steigerung des Gesamtumfanges der gesellschaftlichen Produktion in einem Bereich kaum eine Rolle, wenn modernste Technik durch ein Dutzend oder mehr Kapitale eingeführt wurde; sie erhöhte sich dadurch nicht in nennenswertem Umfange. Als Fall der Profitrate wurde dieser Prozeß erst dann fühlbar, wenn die neue Technik zur allgemeinen technologischen Grundlage der Produktion zu werden begann. Heute ist der Vergesellschaftungsgrad der Produktion aber schon so weit fortgeschritten und gleichzeitig der Sprung in der Ausdehnung der Kapazitäten so groß, daß derselbe Prozeß eine völlige Umwälzung der Marktverhältnisse herbeiführen kann. Es bestehen daher heute schon von vornherein Schwierigkeiten, bei der Einführung neuer Technik die Produktions- und Marktentwicklung einigermaßen in Übereinstimmung zu halten. Dieses Problem ist im Rahmen einzelner Konzerne in ihren jeweils gegebenen Größenordnungen kaum mehr zu lösen. Auch die herkömmlichen Produktions- und Preisabsprachen eines ganzen monopolisierten Zweiges helfen hier nicht mehr. Bei breiter Anwendung der modernen Technik bricht die Marktbeherrschung durch das Monopol zusammen. Es entstehen Überkapazitäten, die die Marktentwicklung auf Jahre hinaus vorwegnehmen und die Kapitalverwertung verschlechtern oder sogar völlig aufheben können. Eine rationelle Anwendung der modernen Technik ist also nur möglich, wenn der Markt sich rapide ausdehnt - und das ist nur in ausgesprochenen Wachstumszweigen der Fall - oder aber wenn das einzelne Kapital seinen Marktanteil entsprechend sprunghaft auszuweiten vermag - und das dürfte eine Ausnahmeerscheinung sein.

Sieht man einmal von ausgesprochenen Wachstumsindustrien ab, so kann sich ein derartiger Vergesellschaftungsgrad der Produktion heute kaum noch durch die Kapitalakkumulation, sondern meist nur noch durch großangelegte Zentralisationsprozesse des Kapitals herstellen. Diese Prozesse aber sind überaus langwierig, gleichguiltig, in welchen Formen sie sich vollziehen, ob durch Kooperation oder durch die Aufsaugung schwächerer Kapitale. Es sind Kapitalmassen von ungeheurer ökonomischer Kraft, die sich heute gegenüberstehen und sie können einem solchen Prozeß sehr lange Widerstand entgegensetzen. Der Zusammenschluß mehrerer Konzerne oder die Spezialisierung der Produktion berührt wiederum private Interessen und die Stellung der einzelnen Konzerne im Gefüge des Gesamtkapitals; Abhängigkeitsverhältnisse können entstehen, Positionen untergraben werden usw. Die Zentralisation erfolgt daher nur unter sehr starkem Druck, nur dann, wenn andere Wege nicht mehr offenstehen. Daher erfolgt der Zentralisationsprozeß im Verhältnis zu den Erfordernissen der technischen .Entwicklung nur langsam. Für die Entwicklung der Produktivkräfte hat das sehr konkrete Folgen. 
Das Kapitalverhältnis wird zu einer Schranke für den technischen Fortschritt. Zunächst einmal nicht absolut. Produktivere Technik wird zwar eingefuihrt, aber es ist eine Technik, die teils sehr weit hinter dem bereits erreichten Stand der technischen Entwicklung zurïckbleibt. Das Kapital kann den technischen Fortschritt nur in dem Maße praktisch nutzen, in dem es die in ihm liegenden Potenzen zur Profitsteigerung auch tatsächlich zu realisieren vermag. Die Frage des Zwanges zur Einführung moderner Technik lasse ich hier beiseite, weil sich dieser Zwang erst dann ergibt, wenn sie bereits in breiterem Umfange eingefuihrt ist und so die Konkurrenzund Verwertungsbedingungen bestimmt.

Diese Schranke findet sehr greifbar Ausdruck. Im Falle der Eisen- und Stahlindustrie z. B. hätte der Mitte der 50er Jahre einsetzende Investitionszyklus, der den Beginn der technischen Revolution in diesem Bereich kennzeichnet, bei einigermaßen konsequenter Anwendung bereits erprobter moderner technischer Verfahren dazu führen können, daß die Eisen- und Stahlproduktion Anfang der 60er Jahre mit 30 Hochöfen, 45 Siemens-Martin-öfen und 13 Konvertern hätte erzielt werden können. In Betrieb waren aber 118 Hochöfen, 145 SM-Öfen und 70 Konverter. Die Potenzen der technischen Entwicklung waren also nur zu etwa 25 Prozent ausgeschöpft worden. Derartige Prozesse sind in allen Industriezweigen, mit Ausnahme der Wachstumsindustrien und für alle Investitionszyklen seit Ende des II. Weltkrieges nachzuweisen.

Die Kapitaleigentumsstrukturen passen sich also nicht den Erfordernissen der technischen Entwicklung an, sondern umgekehrt, die technische Entwicklung wird dem Rahmen der Kapitaleigentumsstrukturen angepaßt. Erst dieser relativ beschränkte Prozeß technischen Fortschritts führt dann zu den Konflikten, die die Kapitaleigentumsstrukturen aufbrechen und die Zentralisationsprozesse des Kapitals auslösen.

Durch diese spezifische Form des technischen Fortschritts entsteht für das Kaf ital eine Reihe von Problemen. Es entwickelt die Produktivkräfte nur dann, wenn es dadurch einen Extraprofit erzielen kann. Das schließt aber ein, daß die mit Hilfe der neuen Technik erzielbare Profitsteigenung größer sein muß als die Vernichtung des noch in den alten, $\mathrm{zu}$ erneuernden Anlagen gebundenen Wertes. Gerade durch diese Form des technischen Fortschritts wird aber nicht nur die Lebensdauer der Anlagen verkürzt, über das Maß hinaus, das an sich schon durch das hohe Tempo der wissenschaftlich-technischen Entwicklung gegeben ist. Gleichzeitig wächst auch noch mit jeder Stufe in diesem Prozeß der Umfang des eingesetzten fixen Kapitals, d.h. es wächst der Umfang der Kapitalvernichtung, der dem Fortschritt der Produktivkräfte entgegensteht.

An sich, gesellschaftlich betrachtet, bildet die Kapitalvernichtung, trotz des schnell wachsenden Umfanges des fixen Kapitals, kein gravierendes Problem. Die moderne Technik wird durch eine so sprunghafte Steigerung der Arbeitsproduktivität gekennzeichnet, daß sich mit ihrer Hilfe auch Kapitalvernichtung großen Umfanges ausgleichen läßt. Aber das Kapital kann diese Potenzen der Profitsteigerung nicht ausschöpfen. Daher hat sich diese Schranke für den technischen Fortschritt im Laufe der Nachkriegsentwicklung zusehends versteift und zwar in einem Maße, daß staatliche Eingriffe notwendig wurden, um sie zu durchbrechen und den kontinuier- 
lichen Ablauf des Prozesses zu ermöglichen. In der Tat wurde die staatliche Regulierung der volkswirtschaftlichen Investitionsprozesse, die vorher hauptsächlich über die direkten staatlichen Investitionen erfolgte, seit Mitte der 50er Jahre mehr und mehr mit den Investitionsprozessen des privaten Kapitals verknüpt. Durch Abschreibungserleichterungen und andere Maßnahmen wurde die Kapiialvernichtung von der Ebene des privaten Kapitals auf die der Gesellschaft verlagert. Natürlich beschränkten sich die staatlichen Maßnahmen nicht auf die steuerliche Begünstigung privater Investitionen. Jede staatlich gesetzte Bedingung, die die Kapitalverwertung beeinflußt oder die auf die Marktausdehnung gerichtet ist und auf diese Weise den Spielraum für die rentable Anwendung moderner Technik erweitert, dient auch der Durchbrechung dieser Schranke für die Entwicklung der Produktivkräfte. Aber diese zunehmende Verknüpfung der staatlichen Eingriffe mit den privaten Investitionsprozessen zeigt besonders deutlich, in welchem Maße die Produktivkraftentwicklung und damit auch der kontinuierliche Ablauf der Investitionsprozesse auf das Kapitalverhältnis als Schranke stieß.

Das Kapitalverhältnis wird aber auch zunehmend zu einer absoluten Schranke für die Entwicklung der Produktivkräfte. Immer mehr Bereiche brechen aus der Sphäre der Bewegung des privaten Kapitals aus und müssen gesellschaftlich entwickelt werden. Ich meine damit nicht nur jene allgemeinen Bedingungen gesellschaftlicher Produktion, die nur gesellschaftlich zu schaffen und zu unterhalten sind, also Straßen usw., obwohl man darauf hinweisen muß, daß der Umfang dieser Bereiche und noch mehr ihre Bedeutung für die Kapitalverwertung mit im Laufe der Entwicklung und bedingt durch den steigenden Vergesellschaftungsgrad ganz erheblich zugenommen haben. Auch sollte man überdenken, inwieweit derartige Bereiche ihren Charakter als allgemeine Bedingung der gesellschaftlichen Produktion überhaupt erst erlangt haben, weil die Stufenleiter und allgemeine Verflechtung der Produktion in so hohem Grade gestiegen sind. Die industrielle Wasserversorgung, beispielsweise, gehörte früher in die Sphäre des privaten Kapitals. Heute hat sie sich zu einer gesellschaftlichen Sphäre erweitert, über die reine Wasserversorgung hinaus bis hin zur Regulierung des Wasserhaushalts - aber eben wegen der Entwicklung der Produktion - und sie ist für die Verwertung des privaten Kapitals ein nicht zu unterschätzender Faktor geworden.

Es ist aber auch überhaupt zu beobachten, daß immer mehr Bereiche aus dem Rahmen der Kapitalverwertung ausbrechen und durch das Kapital daher auch überhaupt nicht mehr oder nur noch unzulänglich entwickelt werden können. Charakteristisch für diese Zweige ist es, daß in ihnen der gesellschaftliche Charakter der Produktion so sprunghaft steigt oder von vornherein so hoch ist, daß es gewisse Zeit dauert, ehe sie mit Produktion und Absatz in das Gefüge des gesellschaftlichen Produktionsorganismus integriert sind. Hohe Kapitalfixierung, überdurchschnittlich lange Umschlagszeiten und Unsicherheit der Kapitalverwertung oder überhaupt des Rückflusses der Mittel kennzeichnen diese Bereiche.

Schon der erste, von seiner allgemeinen Bedeutung her wesentliche staatsmonopolistische Eingriff in Deutschland wurde durch dieses Phänomen verursacht. Ich meine den Aufbau der Elektroenergieversorgung. Um diese Energiequelle für die Produktion nutzbar zu machen, mußte der Strom so billig erzeugt und verteilt 
werden können, daß er die herkömmlichen Energiesysteme zu verdrängen vermochte. Das war nur über kostspielige Großanlagen möglich, für die wiederum der Markt fehlte. Der Profit als Maßstab der Entwicklung versagte hier vollkommen. Der Staat mußte eingreifen. Er baute bzw. finanzierte die Anlagen oder er garantierte den Absatz.

Heute gibt es eine Vielzahl solcher Bereiche. Als Beispiel kann man auf die Nutzung der Kernenergie, bestimmte Verkehrs- und Nachrichtenmittel, bestimmte Bereiche der Elektrotechnik usw. verweisen. Auch der Komplex Forschung und Entwicklung kann hier eingeordnet werden. Ebenso solche Bereiche wie der Steinkohlebergbau, die im Zuge der technischen Revolution an relativer Bedeutung verlieren und deren Neueingliederung in das Gefüge der gesellschaftlichen Produktion, für die sie nach wie vor unentbehrlich sind, im Rahmen des privaten Kapitals nicht mehr möglich ist. Die Entwicklung aller dieser Bereiche muß, direkt oder indirekt, vom Staat übernommen werden.

Bevor ich diesen Komplex abschließe, ist es vielleicht doch angebracht, eine Vorstellung von den Größenordnungen staatlicher Eingriffe zu vermitteln. Wenn man nur die Subventionen und die geschätzten steuerlichen Vergünstigungen nimmt, so waren sie 1965 fast so groß und 1969 etwa eineinhalbmal so groß wie die gesamten Investitionen der verarbeitenden Industrie. Zählt man noch die direkten staatlichen Investitionen hinzu - und damit hat man noch keineswegs alle staatlichen Eingriffe erfaßt - so ergeben sich Größenordnungen, die rund 30 bzw. 36 Prozent der gesamtvolkswirtschaftlichen Investitionen entsprechen. Derartige Größenordnungen sind in Aufschwungszeiten und im Frieden nie zuvor erreicht worden.

Der kapitalistische Grundwiderspruch wird heute also schon unmittelbar wirksam. Das Kapitalverhältnis tritt in der Tat als Schranke der Entwicklung der Produktivkräfte hervor; und nicht nur der Entwicklung der Produktivkräfte, sondern der gesellschaftlichen Entwicklung überhaupt. Ich habe die staatlichen Eingriffe hier unmittelbar in Zusammenhang mit der Entwicklung der Produktivkräfte gebracht, weil in dieser Wirkungsform des Grundkonflikts auch die Problematik der Ausbildung des staatsmonopolistischen Kapitalismus und die historische Begrenztheit, der Übergangscharakter des gegenwärtigen Kapitalismus, voll zum Ausdruck kommen. Aber sie dienen natürlich ebenso der Regulierung der zyklischen Produktionsbewegung bzw. anderer Krisenformen, die heute ein neues Gewicht erlangt haben, beispielsweise der Regionalkrisen usw. Die ganze Problematik ist also außerordentlich komplex. Es ging hier aber auch nur darum, zu zeigen, daß die Einbeziehung des bürgerlichen Staates in die Wirtschaft notwendig geworden ist, um die Anwendung der hochgradig gesellschaftlichen Produktivkräfte auf privatkapitalistischer Basis überhaupt noch möglich zu machen. Aus der Konfliktentfaltung erklärt sich die Notwendigkeit, die Gesetzmäßigkeit der Ausbildung des staatsmonopolistischen Kapitalismus. Er ist eine notwendige Anpassung der kapitalistischen Produktionsverhältnisse an den Vergesellschaftungsgrad der Produktion, das gesetzmäßige Dodukt einer unter kapitalistischen Bedingungen verlaufenden Vergesellschaftung roduktion in den heutigen Ausmaßen, d.h. des Drucks der systemimmanenten reprüche und seiner sich daraus ergebenden ökonomischen und politischen sectit. 
Es ist also eine unzulässige Vereinfachung, wenn man den Begriff des staatsmonopolistischen Kapitalismus lediglich dahingehend definiert, daß der bürgerliche Staat von den Monopolen benutzt würde, um ihnen Höchstprofite zuzuschanzen. Richtig ist diese Definition nur, soweit sie den staatsmonopolistischen Kapitalismus als zugespitzte Entwicklungsform des kapitalistischen Ausbeutungsverhältnisses charakterisiert. Das ist in der Tat ein entscheidender Wesenszug; und darauf möchte ich jetzt noch eingehen.

Grundlage der Ausbeutung ist das private Eigentum an den Produktionsmitteln. Aus ihm leitet sich das Recht bzw. die Macht der Eigentümer zur Aneignung des in der gesellschaftlichen Produktion erzeugten Mehrwerts bzw. Profits ab. (Die Unterscheidung zwischen Mehrwert und Profit ist in unserem Zusammenhang ohne Belang.) Das gilt grundsätzlich auch noch heute. Dennoch sind in dem Prozeß der Aneignung und Verteilung des Profits Veränderungen qualitativer Art eingetreten.

Im einfachen Kapitalismus wurde der auf das einzelne Kapital entfallende Teil des gesellschaftlichen Gesamtprofits durch das Gesetz der Durchschnittsprofitrate bestimmt. Die Konkurrenz reduzierte den Profitanteil eines jeden Einzelkapitals letztlich auf den, seinem relativen Anteil am Gesamtkapital entsprechenden Profitteil. Mit der Entstehung des Monopols kommt in diesen Prozeß ein neues Moment; das der ökonomischen Macht. Kraft seiner ökonomischen Machtstellung ist das Monopol in der Lage, die Konkurrenz in bestimmter Hinsicht auszuschalten - obwohl es sie nicht aufzuheben vermag - dadurch das Gesetz des Durchschnittsprofits zu durchbrechen und sich einen zusätzlichen Profitteil, über den Durchschnittsprofit hinaus, anzueignen. (Über die Frage, ob und wie sich das Gesetz des Durchschnittsprofits dennoch durchsetzt, gibt es unter Marxisten unterschiedliche Auffassungen und es würde zu weit führen, hier meine eigene darzulegen.) Durch das Monopol wird aber nicht nur der Verteilungsmechanismus des Profits verändert. Das Ausbeutungsverhältnis selbst witd erweitert, denn das Monopol kann durch seine Machtstellung Wertteile aus Produktionsbereichen an sich ziehen, die vorher nicht der Ausbeutung unterlagen; z. B. aus der einfachen Warenproduktion.

Beim staatsmonopolistischen Kapitalismus liegen die Dinge nicht so einfach. Das Monopol ist eine Entwicklungsform des Privatkapitals. Der Staat ist ein zentralisiertes Machtinstrument des Gesamtkapitals. Das Privatkapital ist ein Element der Produktionsverhältnisse, der Basis, der Staat ein Element des Überbaues. Der staatsmonopolistische Kapitalismus kann daher nicht einfach eine Entwicklungsform des Privatkapitals sein. Wenn man vom staatsmonopolistischen Kapitalismus als einer Entwicklungsform des Kapitalverhältnisses sprechen kann, so deshalb, weil der Staat neue ökonomische Funktionen ausübt, mit denen er auf die Bedingungen der Profitaneignung durch das Privatkapital und ebenso auf die Bedingungen der Profitproduktion und der Profitrealisierung einwirkt, mit denen er direkt in den kapitalistischen Mechanismus der Profitaneignung und -verteilung einbezogen wird.

Im vormonopolistischen Kapitalismus waren die ökonomischen Funktionen des Staates vergleichsweise gering. Er hatte für dis allgemeinen Bedingungen der Produktion zu sorgen, die nur gesellschaftlich zu schaffen waren. Er stellte $e^{:}$ gewisse ökonomische Größe dar, einen gewissen Markt, war aber in dieser R' hung weder für die Bewegung des Kapitals noch für die Entwicklung der Pro? 
kräfte von wesentlicher Bedeutung. Für die Kapitalverwertung spielte er eigentlich nur insofern eine größere Rolle - und auch das nur, wenn man die Entwicklung des Kapitalismus in seiner konkreten, nationalen Gestalt, nicht aber die als gesellschaftliches System im Auge hat - als er das nationale Kapital gegen ausländische Konkurrenz abschirmte. Schon jetzt kann man allerdings die charakteristischen Merkmale der Ausnutzungsmöglichkeit staatlicher Macht durch das Kapital erkennen. Der Staat kann im Aneignungs- und Umverteilungsprozeß von Nationaleinkommen alle Klassen und Schichten der Bevölkerung und alle Bereiche der Wirtschaft erfassen; und zwar unabhängig von den sich aus den gesellschaftlichen Zusammenhängen der Produktion oder ökonomischen Einzugsbereichen ergebenden Beziehungen, die die Macht des Monopols in dieser Hinsicht begrenzen. Der Teil des Nationaleinkommens, den der Staat an sich zieht, unterliegt in seinem Einsatz nicht dem Zwang zur Kapitalverwertung; beim Monopol ist das aber der Fall. Schließlich: wenn der Staat Bedingungen in der Wirtschaft setzt, gleich welcher Art, die auf die Bewegung des Kapitals gerichtet sind, so handelt es sich dabei um Monopolbedingungen, um Bedingungen, die gegen das ungehemmte Wirken des Konkurrenzmechanismus gerichtet sind und die eine Unverteilung von Nationaleinkommen bewirken, d. h. eine kraft staatlicher Gewalt bewirkte Verteilung gegen die sich aus dem eigentlichen inneren Mechanismus des Kapitalismus ergebende Verteilung. Staatliche Macht kann also im Prozeß der Profitaneignung und -verteilung als zusätzliches Moment in Erscheinung treten.

Betrachtet man nun die Rolle des Staates in der Ökonomie heute, so muß man feststellen, daß sich im Laufe der Entwicklung eine qualitative Veränderung vollzogen hat. Der Staat ist unmittelbar in den Reproduktionsprozeß und in den Verwertungsprozeß des Kapitals einbezogen worden. Er entwickelt bestimmte, für den Gesamtreproduktionsprozeß des Kapitals und für seine Verwertung unentbehrliche Bereiche und zwar weit über den Rahmen der allgemeinen Bedingungen der gesellschaftlichen Produktion hinaus. Er übernimmt Aufgaben der Mobilisierung der für die Akkumulation unter den heutigen Bedingungen notwendigen Kapitalmassen. Er greift in den Regulierungsmechanismus der gesellschaftlichen Produktion ein und zwar sowohl im Hinblick auf die Gestaltung der volkswirtschaftlichen Strukturen, als auch im Hinblick auf das Verhältnis zwischen Produktion und Markt und auf das gesamtvolkswirtschaftliche Wachstum. Er ist also in den Reproduktionsprozeß des gesellschaftlichen Gesamtkapitals direkt einbezogen worden und er überzieht die Wirtschaft mit einem Netz von Maßnahmen, mit denen er in den Prozeß der Profitaneignung und -verteilung eingreift. Es gibt heute faktisch keinen einzigen volkswirtschaftlichen Bereich mehr, in dem der Staat nicht in der einen oder anderen Form die sich aus dem inneren Mechanismus des Kapitalismus ergebende Verteilung des Nationaleinkommens durchbricht und so, kraft staatlicher Macht, Verwertungsbedingungen für das Kapital setzt. Vom Standpunkt des Kapitalverhältnisses aus gesehen, ist also ein neues Moment in den Mechanismus der Aneignung und Verteilung des Profits einbezogen worden, das der außerökonomischen Gewalt. Der Staat fungiert heute unmittelbar im Ausbeutungsprozeß. Die ökonomische Macht des Monopols in diesem Prozeß wird durch staatlich gesetzte Monopolbedingungen ergänzt. 
Auch dies ist nicht schematisch aufzufassen, als einfache Aneignung und Umverteilung von Nationaleinkommen durch den Staat zugunsten der Monopole. Das ist nur die allgemeine Grundlage. Indem der Staat Verwertungsbedingungen für das Kapital setzt und so die Schranken für die Entwicklung der Produktivkräfte durch das Monopolkapital durchbricht, beeinflußt er zugleich die Bedingungen der Profitproduktion beim Monopol selbst. Wenn der Staat Marktbedingungen setzt, beeinflußt er die Bedingungen der Profitrealisierung und erweitert er über diese wiederum die Möglichkeiten der Produktivkraftentwicklung für das Privatkapital und folglich die Bedignungen der Profitproduktion. Die ganze Bewegung des Kapitals vollzieht sich also nach wie vor im Selbstlauf, soweit dies uberhaupt noch möglich ist, angetrieben von den inneren Gesetzmäßigkeiten des Kapitals. Aber die staatlichen Eingriffe bilden die Grundlage für diesen Selbstlanf, ermöglichen ihn, indem sie die stets von neuem entstehenden Schranken stets von neuem durchbrechen. Sie schaffen den Spielraum, der die Bewegung der gesellschaftlichen Produktion als kapitalistische Produktion überhaupt noch ermöglicht.

Die Schwierigkeiten in der Erkenntnis des staatsmonopolistischen Kapitalismus als höhere Form der Monopolisienung liegen in der besonderen Stellung des Staates im System einer Produktionsweise. Die staatlichen Eingriffe sind in der Regel allgemeiner Natur. Sie sind weniger auf die bessere Verwertung dieses oder jenes Monopols gerichtet - obwohl auch das durchaus eine Rolle spielen kann - als vielmehr auf die Bewegung und Entwicklung der gesellschaftlichen Produktion insgesamt - aber eben auf ihre Bewegung in diesem ganz spezifischen Rahmen, der durch das Kapitalverhältnis geprägt ist und in dem sich die Bewegung nur über den Profit und die Beeinflussung der Profitbedingungen vollziehen kann. Die Grenzen, die sich aus diesem engen Rahmen für die Entwicklung auf einer bestimmten Stufenleiter der Vergesellschaftung der Produktion ergeben, können, auf kapitalistischer Basis, nur über die Monopolisierung durchbrochen werden. Andere Formen der notwendig werdenden Veränderungen im Prozeß der Aneignung und Verteilung des Profits gibt es nicht. Das Monopol ist aber zugleich die letztmögliche Entwicklungsform des privaten Kapitalverhältnisses. Eine darüber hinausgehende Möglichkeit des Eingriffs in diesen Mechanismus ist nur durch die Anwendung staatlicher Machtmittel gegeben.

Durch die besondere Stellung des Staates ergibt sich natürlich ein Moment, das geeignet ist, das Wesen des staatsmonopolistischen Kapitalismus bis zu einem gewissen Grade zu verbergen. Staatliche Eingriffe kömien sich sowohl zugunsten monopolisierter wie nichtmonopolisierter Bereiche auswirken, sie können bestimmte nichtmonopolisierte Bereiche begünstigen und gleichzeitig gegen die Interessen bestimmter monopolisierter Zweige gerichtet sein. Der staatsmonopolistische Kapitalismus ist in seinen Oberflächenerscheinungen durchaus widersprïchlich. Der Sichleier verschwindet jedoch, wenn man die Ursachen und die auf die Bewegung des Kapitals begrenzte Funktion des staatlichen Wirkens in der Ökonomie bei der Betrachtung berïcksichtigt. 
Zunächst einmal muß man die allgemeine Basis bedenken, auf die die staatlichen Eingriffe gerichtet sind. Die Produktion ist heute hochgradig gesellschaftlich. In der Industrie beschäftigten 1967 rund 12 Prozent aller Unternehmen rund 71 Prozent der Arbeitskräfte und sie bestritten rund 80 Prozent des Umsatzes. Berücksichtigt man die eigentumsmäßige Verflechtung dieser Unternehmen untereinander, so verringert sich der Prozentsatz der Unternehmen noch weiter. In den wichtigsten anderen volkswirtschaftlichen Bereichen besteht ein ähnlicher $\mathrm{G}$ rad der Konzentration von Produktion und Kapital. Die gesellschaftliche Arbeitsteilung ist heute überdies so weitgehend entwickelt, die einzelnen Unternehmen sind so hochgradig spezialisiert, die ökonomischen Beziehungen zwischen den verschiedenen Produktionszweigen und volkswirtschaftlichen Bereichen sind so eng, daß auch die nichtmonopolisierten Kapitale entweder in die Produktionszusammenhänge der monopolisierten Zweige einbezogen sind oder in irgendeiner Weise vom Monopol erfaßt werden können. D. h. die gesamte Wirtschaft ist heute weitgehend monopolisiert und das Monopol ist die allgemeine Grundlage, die die Verteilung des Profits bestimmt. In einer solchen Wirtschaft, die faktisch mehr und mehr einem gesamtgesellschaftlichen Produktionsorganismus gleicht, dessen Entwicklung und Produktivität weitgehend von der Entwicklung, Produktivität und Proportionalität seiner einzelnen Teile abhängt, in der die Verwertung des gesellschaftlichen Gesamtkapitals, die Gesamtmasse des Profits, folglich in stärkstem Maße von dem technischen Stand und der optimalen Struktur des Gesamtorganismus bestimmt wird, in einer solchen Wirtschaft schlägt sich jede staatliche Einwirkung, die auf die Bewegung des Kapitals gerichtet ist, letztlich als Einwirkung zugunsten des Monopolkapitals nieder. Indirekt, weil das Monopol in der Lage ist, Profit aus nichtmonopolisierten Zweigen an sich zu ziehen. Direkt, weil jene Unternehmen, die rund 71 Prozent der Arbeitskräfte beschäftigen und rund $80 \%$ des Umsatzes tätigen, ohnehin in erster Linie von der Entwicklung des Transport- und Nachrichtenwesens, der Energieversorgung, der Forschung, der Exportförderung, der Staatsausgaben generell und selbst der staatlichen Wohnungsbauförderung profitieren müssen; allein schon von den Größenordnungen und der hohen gesellschaftlichen Stufenleiter ihrer Produktion her.

Zum anderen kann sich die staatliche Monopolisierung garnicht auf einzelne Maßnahmen oder einzelne Bereiche beschränken. Der Vergesellschaftungsgrad der Produktion ist so hoch und die sich dåraus ergebende Schärfe der systemimmanenten Konflikte so groß, daß sich daraus in zunehmendem Maße die Notwendigkeit ergibt, auch in kapitalistischem Rahmen Formen gesellschaftlicher Organisation und Regulierung der Produktion zu finden. Das ist nicht nur eine Frage der sozialen und politischen Konfliktentfaltung, sondern auch schon der Kapitalbewegung selbst. Die Zusammenhänge sind so komplex und von so unmittelbarem Einfluß, daß die staatliche Monopolisierung die Gesamtheit der ökonomischen Bedingungen berücksichtigen muß. Anders kann sie ihre Funktion, Raum für die Bewegung der gesellschaftlichen Produktion als kapitalistische Produktion zu schaffen, nicht erfuillen.

Die Ausrichtung staatlicher Eingriffe auf diese Zielstellung ist jedoch kein bewußter, planmäßiger Vorgang. Es handelt sich um eine objektive Notwendigkeit, um Sachzwänge, die selbst Ergebnis der Wechselwirkung verschiedener Prozesse 
sind. Das Verhältnis Staat - Monopol ist kompliziert. Faßt man es dahingehend zusammen, daß der Staat den Interessen des Monopolkapitals untergeordnet wird, so wird es keineswegs in der Vielfalt seiner Beziehungen voll erfaßt; obwohl das Wesentliche der objektiven Tatbestände damit charakterisiert wird. In der Praxis hat sich dieses Verhältnis sicherlich zunächst als Ausnutzung des Staatsapparates im Interesse einzelner Monopole oder Monopolgruppen entwickelt. Der Staatsapparat selber ist aber kein Bestandteil des Monopolapparates, obwohl in vielfältiger Weise, über ökonomische und personelle Zusammenhänge mit ihm verflochten. Reduziert man die Zusammenhänge auf das Wesentliche, so ist der Staat wohl ein Instrument des Monopolkapitals. Aber er ist zugleich auch eine Sphäre der Konkurrenz der Monopole um die Ausnutzung der staatlichen Macht zu ihren eigenen Gunsten, zugunsten ihrer besonderen, spezifischen Interessen. In der Praxis ist es also eine Frage der Stärkeverhältnisse, der Interessengemeinschaften oder -kollisionen und der jeweils gegebenen allgemeinen ökonomischen und politischen Bedingungen, in welcher Richtung die staatsmonopolistischen Maßnahmen in besonderem Maße wirken. Damit sind bei weitem noch nicht alle Einflußfaktoren erfaßt; nicht die Eigeninteressen der staatlichen Bürokratie, nicht die politischen Einflüsse, nicht die von der Systemsicherung her gegebenen Faktoren usw.

Die auf das Gesamtfunktionieren des Systems gerichtete Aufgäbe der staatlichen Monopolisierung setzt sich also als Ergebnis dieser sehr konkreten und sehr unterschiedlichen, teilweise antagonistischen Einflüsse durch. Aus der objektiven Ausrichtung der staatlichen Monopolisierung einerseits und der Vielfalt der konkreten Bedingungen, unter denen sie sich entfaltet, andererseits, erklärt sich die Vielfalt und oft scheinbare Widersprüchlichkeit der staatsmonopolistischen Erscheinungen, erklärt es sich, daß staatliche Eingriffe durchaus auch gegen die Interessen einzelner Monopole oder Monopolgruppen gerichtet sein können - und sogar gerichtet sein müssen, wenn diese das Gesamtfunktionieren des Systems untergraben, $\mathrm{daß}$ sie auch nichtmonopolisierte Bereiche begünstigen können und daß von Monopolen geforderte Maßnahmen oft überhaupt nicht zustande kommen oder durchgesetzte wirkungslos verpuffen. Die Prozesse sind also äußerst vielschichtig. Das ändert jedoch nichts am Wesen dieser staatlichen Funktion.

In meinen Darlegungen habe ich eine Fülle von Problemen nicht berücksichtigen können. Nicht einmal die Ursachen der Ausbildung des staatsmonopolistischen Kapitalismus konnten in ihrer ganzen Vielfalt und Wechselbezogenheit dargestellt werden. Mir kam es jedoch vor allem darauf an, zu zeigen, daß der staatsmonopolistische Kapitalismus nicht allein als Umverteilung von Nationaleinkommen durch den Staat zugunsten der Monopole zu begreifen ist, sondern daß die Einbeziehung des Staates in die Ökonomie ein gesetzmäßiger Prozeß ist, der sich aus den inneren Konflikten dieser Produktionsweise notwendig ergibt, um die Bewegung der gesellschaftlichen Produktion als kapitalistische Produktion zu ermöglichen. Gleichzeitig wollte ich die Potenzen dieses Systems andeuten, die darin liegen, daß es zur Basis einer weiteren Entwicklung der Produktivkräfte auf kapitalistischer Grundlage wird, und seine Grenzen, die darin liegen, daß es die Produktionsverhältnisse nicht aufhebt und die Vergesellschaftung der Produktion weiter vorantreibt und so zugleich die Entfaltung der Konflikte, die die bewußte, vorausschauende gesellschaft- 
liche Organisation und Regulienung der Produktion als Bedingung für die Bewältigung der Entwicklungsprobleme der modernen Gesellschaft zu einer objektiven Notwendigkeit machen.

Literaturangaben

1. R. Gändel, H. Heininger, P. Hess, K. Zieschang, Zur Theorie des staatsmonopolistischen Kapitalismus, Akademie-Verlag, Berlin 1971

2. Kurt Zieschang, Ursachen und Wesen des staatsmonopolistischen Kapitalismus, Wirtschaftswissenschaft, Berlin, H. 2/1973 